International Journal of Pure and Applied Mathematics

Volume 112 No. 1 2017, 189-201

ISSN: 1311-8080 (printed version); ISSN: 1314-3395 (on-line version)

url: http://www.ijpam.eu

doi: 10.12732 /jpam.v112i1.15

\title{
HYERS-ULAM STABILITY OF QUADRATIC FUNCTIONAL EQUATIONS ON DIVISIBLE SQUARE-SYMMETRIC GROUPOID
}

\author{
Gwang Hui Kim ${ }^{1}$, Hwan-Yong Shin ${ }^{2} \S$ \\ ${ }^{1}$ Department of Mathematics \\ Kangnam Universaty \\ Yongin, Gyeonggi, 16979, REPUBLIC OF KOREA \\ ${ }^{2}$ Department of Mathematics \\ Chungnam National University \\ 99 Daehangno, Yuseong-gu, Daejeon, 34134, REPUBLIC OF KOREA
}

\begin{abstract}
Let $(X, \diamond)$ be a divisible square-symmetric groupoid, and $(Y, *, d)$ a complete metric divisible square-symmetric groupoid. In this paper, we obtain the Hyers-Ulam stability problem of functional inequality $d\left(f(x \diamond y) * f\left(x \diamond y^{-1}\right), \sigma_{*}(f(x) * f(y))\right) \leq \varepsilon(x, y)$ for approximate mapping $f: X \rightarrow Y$ of functional equation $h(x \diamond y) * h\left(x \diamond y^{-1}\right)=\sigma_{*}(h(x) * h(y))$ differing by $\varepsilon: X^{2} \rightarrow[0, \infty)$.
\end{abstract}

AMS Subject Classification: 39B52, 39B72, 47H09

Key Words: Hyers-Ulam stability, quadratic functional equation, square-symmetric groupoid, fixed point theorem

\section{Introduction}

In 1940, S.M. Ulam [19] proposed the Hyers-Ulam stability problem for functional equations concerning the stability of group homomorphisms : Under what condition does there exist a homomorphism near an approximately homomorphism?

Received: $\quad$ September 28, 2016

Revised: $\quad$ October 16, 2016

Published: January 26, 2017

$\S_{\text {Correspondence author }}$ (c) 2017 Academic Publications, Ltd. url: www.acadpubl.eu 
In 1941, D.H. Hyers [9] considered the case of approximate additive mappings with the Cauchy difference controlled by a positive constant in Banach spaces. Thereafter we usually say that the equation $E_{1}(h)=E_{2}(h)$ has the Hyers-Ulam stability if for an approximate solution $f$ of this equation, i.e., for a function $f$ with $\left|E_{1}(f)-E_{2}(f)\right| \leq \delta$, there exists a function $g$ such that $E_{1}(g)=E_{2}(g)$ and $|f(x)-g(x)| \leq \varepsilon$.

D.G. Bourgin [3] and T. Aoki [2] treated this problem for approximate additive mappings controlled by unbounded function. In [17], Th. M. Rassias provided a generalization of Hyers' theorem for linear mappings which allows the Cauchy difference to be unbounded. In 1994, P. Găvruta [8] generalized these theorems for approximate additive mappings controlled by the unbounded Cauchy difference with regular conditions. During the last three decades a number of papers and research monographs have been published on various generalizations and applications of the Hyers-Ulam stability and generalized Hyers-Ulam stability to a number of functional equations and mappings $[1,5$, $7,10,16]$.

The functional equation $f(x+y)+f(x-y)=2 f(x)+2 f(y)$ is said to von-Nuemann functional equation. In particular, every solution of the vonNuemann functional equation is said to be a quadratic function. The HyersUlam stability of von-Nuemann functional equation was proved by Skof [18] for mapping $f: E_{1} \rightarrow E_{2}$, where $E_{1}$ is a normed space and $E_{2}$ is a Banach space. For more information of stability of von-Neumann functional equation, we refer $[4,6,12,15]$.

Let $(X, \diamond),(Y, *)$ be groupoids with binary operations. If the binary operation $\diamond$ satisfies the following identity,

$$
(x \diamond y) \diamond(x \diamond y)=(x \diamond x) \diamond(y \diamond y) \quad(x, y \in X),
$$

the operation $\diamond$ will be called square-symmetric. Note that the square-symmetric of $\diamond$ implies that $\sigma_{\diamond}(x):=x \diamond x$ is an endomorphism. Thus we remark that

$$
\sigma_{\diamond}^{n}(x) \diamond \sigma_{\diamond}^{n}(y)=\sigma_{\diamond}^{n}(x \diamond y)
$$

for all $n \in \mathbb{N}$. It is an crucial relation when the mapping $\sigma_{\diamond}^{n}$ is an automorphism of $(X, \diamond)$, that is, when $\sigma_{\diamond}$ is bijective. Then, for each $y \in X$ the equation $\sigma_{\diamond}(x)=y$ can be solved uniquely with respect to $x \in X$. Such an element $x$ is denoted by $x=\sigma_{\diamond}^{-1}(y)$. Then it is easy to see that $\sigma_{\diamond}^{-1}: X \rightarrow X$ is an endomorphism. A binary operation $\diamond$ such that $\sigma_{\diamond}$ is an automorphism of $(X, \diamond)$ is called divisible, the corresponding groupoid is said to be a divisible groupoid. 
We remember that the Lipschitz modulus $\operatorname{Lip}(\phi)$ for a function $\phi: Y \rightarrow Y$ on the space $Y$ with metric $d$ defined by

$$
\operatorname{Lip}(\phi):=\sup \left\{\frac{d(\phi(x) \cdot \phi(y))}{d(x, y)} \mid x, y \in Y, x \neq y\right\} .
$$

Then it follows that $\operatorname{Lip}(\phi \circ \psi) \leq \operatorname{Lip}(\phi) \operatorname{Lip}(\psi)$.

Over the setting of square-symmetric groupoid, G.H. Kim [11] obtain the Hyers-Ulam stability of the following functional inequality $d(f(x \diamond y), f(x) *$ $f(y)) \leq \varepsilon(x, y)$ for approximate mapping $f: X \rightarrow Y$ of functional equation $h(x \diamond y)=h(x) * h(y)$ differing by $\varepsilon: X^{2} \rightarrow[0, \infty)$.

This paper aims to investigate the Hyers-Ulam stability for the following functional equation of $f: X \rightarrow Y$ :

$$
f(x \diamond y) * f\left(x \diamond y^{-1}\right)=\sigma_{*}(f(x) * f(y)) \quad(x, y \in X) .
$$

A particular case of (1) is the von-Nuemann functional equation when $X, Y$ are semigroups with the operation + . Thus we will have the generalized stability results of von-Neumann functional equation.

\section{The Hyers-Ulam Stability of Functional Equation (1) via Direct Method}

Theorem 1. Let $(X, \diamond)$ be a divisible square-symmetric groupoid and let $(Y, *, d)$ be a complete metric divisible square-symmetric groupoid. Assume that a mapping $f: X \rightarrow Y$ satisfies

$$
d\left(f(x \diamond y) * f\left(x \diamond y^{-1}\right), \sigma_{*}(f(x) * f(y))\right) \leq \varepsilon(x, y)
$$

for all $x, y \in X$ with $f\left(i d_{X}\right)=i d_{Y}$ and $\varepsilon: X^{2} \rightarrow[0, \infty)$ is a mapping such that

$$
L_{1}(x, y):=\sum_{n=0}^{\infty} \operatorname{Lip}\left(\sigma_{*}^{-2 n}\right) \varepsilon\left(\sigma_{\diamond}^{n}(x), \sigma_{\diamond}^{n}(y)\right)<\infty
$$

for all $x, y \in X$. Then for every $x \in X$,

$$
h(x):=\lim _{n \rightarrow \infty} \sigma_{*}^{-2 n} \circ f \circ \sigma_{\diamond}^{n}(x)
$$

converges and $h: X \rightarrow Y$ is the solution of (1) such that

$$
d(f(x), h(x)) \leq \operatorname{Lip}\left(\sigma_{*}^{-2}\right) L_{1}(x, x)
$$


for all $x \in X$.

Moreover, if $\left\{\operatorname{Lip}\left(\sigma_{*}^{-2 n}\right) \operatorname{Lip}\left(\sigma_{*}^{2 n}\right)\right\}_{n \geq 1}$ is bounded then $h$ is the only solution of (1) such that the mapping $x \rightarrow d(f(x), h(x))$ is bounded with bound $\operatorname{Lip}\left({\sigma_{*}^{-2}}^{-2} L(x, x)\right.$.

Proof. Define $f_{n}: X \rightarrow Y$ by

$$
f_{0}:=f, \quad f_{n}:=\sigma_{*}^{-2 n} \circ f \circ \sigma_{\diamond}^{n}
$$

for all positive integer $n \in \mathbb{N}$. First, we would like to prove that for each fixed $x \in X$ the sequence $\left\{f_{n}(x)\right\}$ is convergent. Replacing $x$ and $y$ by $\sigma_{\diamond}^{n}(x)$ in equality (2), we get

$$
\begin{aligned}
d\left(f\left(\sigma_{\diamond}^{n}(x) \diamond \sigma_{\diamond}^{n}(x)\right) * f\left(\sigma_{\diamond}^{n}(x) \diamond \sigma_{\diamond}^{-n}(x)\right), \sigma_{*}\left(f\left(\sigma_{\diamond}^{n}(x)\right) *\right.\right. & \left.f\left(\sigma_{\diamond}^{n}(x)\right)\right) \\
& \leq \varepsilon\left(\sigma_{\diamond}^{n}(x), \sigma_{\diamond}^{n}(x)\right),
\end{aligned}
$$

which implies that

$$
d\left(f \circ \sigma_{\diamond}^{n+1}(x), \sigma_{*}^{2} \circ f \circ \sigma_{\diamond}^{n}(x)\right) \leq \varepsilon\left(\sigma_{\diamond}^{n}(x), \sigma_{\diamond}^{n}(x)\right) .
$$

Thus we obtain

$$
\begin{aligned}
d\left(f_{n+1}(x), f_{n}(x)\right) & =d\left(\sigma_{*}^{-2(n+1)} \circ f \circ \sigma_{\diamond}^{n+1}(x), \sigma_{*}^{-2(n+1)} \circ \sigma_{*}^{2}(x) \circ f \circ \sigma_{\diamond}^{n}(x)\right) \\
& \leq \operatorname{Lip}\left(\sigma_{*}^{-2(n+1)}\right) d\left(f \circ \sigma_{\diamond}^{n+1}(x), \sigma_{*}^{2} \circ f \circ \sigma_{\diamond}^{n}(x)\right) \\
& \leq \operatorname{Lip}\left(\sigma_{*}^{-2(n+1)}\right) \varepsilon\left(\sigma_{\diamond}^{n}(x), \sigma_{\diamond}^{n}(x)\right) .
\end{aligned}
$$

It produce the following inequality that for positive integers $m, n$ with $m, n>0$,

$$
\begin{aligned}
d\left(f_{n}(x), f_{m}(x)\right) & \leq \sum_{k=n}^{m-1} d\left(f_{k}(x), f_{k+1}(x)\right) \\
& \leq \sum_{k=n}^{m-1} \operatorname{Lip}\left(\sigma_{*}^{-2(k+1)}\right) \varepsilon\left(\sigma_{\diamond}^{k}(x), \sigma_{\diamond}^{k}(x)\right) \\
& \rightarrow 0 \quad \text { as } n \rightarrow \infty,
\end{aligned}
$$

which shows that the sequence $\left\{f_{n}(x)\right\}$ is Cauchy and thus converges in $Y$. Since $Y$ is complete, we can define a function $h: X \rightarrow Y$ as follows

$$
h(x):=\lim _{n \rightarrow \infty} \sigma_{*}^{-2 n} \circ f \circ \sigma_{\diamond}^{n}(x)
$$


for all $x \in X$. Now, for $n \in \mathbb{N}$, we have

$$
\begin{aligned}
d\left(f_{n}(x), f(x)\right) & \leq \sum_{k=0}^{n-1} d\left(f_{k+1}(x), f_{k}(x)\right) \\
& \leq \sum_{k=0}^{n-1} \operatorname{Lip}\left(\sigma_{*}^{-2(k+1)}\right) \varepsilon\left(\sigma_{\diamond}^{k}(x), \sigma_{\diamond}^{k}(x)\right) \\
& \leq \sum_{k=0}^{\infty} \operatorname{Lip}\left(\sigma_{*}^{-2(k+1)}\right) \varepsilon\left(\sigma_{\diamond}^{k}(x), \sigma_{\diamond}^{k}(x)\right) \\
& \leq \operatorname{Lip}\left(\sigma_{*}^{-2}\right) L_{1}(x, x)
\end{aligned}
$$

for all $x \in X$. Taking $n \rightarrow \infty$, we get (4) for the limit function $h$ of $\left\{f_{n}\right\}$.

Next, we wil prove that $h$ satisfies (1). Replacing $\mathrm{x}$ and $\mathrm{y}$ by $\sigma_{\diamond}^{n}(x)$ and $\sigma_{\diamond}^{n}(y)$ in $(2)$ and using the endomorphism of $\sigma_{\diamond}$, we get

$$
\begin{aligned}
d\left(\left(f \circ \sigma_{\diamond}^{n}(x \diamond y)\right) *\left(f \circ \sigma_{\diamond}^{n}\left(x \diamond y^{-1}\right)\right), \sigma_{*}\left(\left(f \circ \sigma_{\diamond}^{n}(x)\right) *(f \circ\right.\right. & \left.\left.\left.\sigma_{\diamond}^{n}(x)\right)\right)\right) \\
& \leq \varepsilon\left(\sigma_{\diamond}^{n}(x), \sigma_{\diamond}^{n}(y)\right)
\end{aligned}
$$

for all $x, y \in X$. Since $\sigma_{*}$ is an automorphism of $(Y, *), \sigma_{*}^{-2 n}$ is also automorphism for all $n$. Therefore,

$$
\begin{aligned}
& d\left(f_{n}(x \diamond y) * f_{n}\left(x \diamond y^{-1}\right), \sigma_{*}\left(f_{n}(x) * f_{n}(y)\right)\right. \\
& =d\left(\sigma_{*}^{-2 n}\left(\left(f \circ \sigma_{\diamond}^{n}\right)(x \diamond y) *\left(f \circ \sigma_{\diamond}^{n}\right)\left(x \diamond y^{-1}\right)\right),\right. \\
& \left.\quad \sigma_{*}^{-2 n+1}\left(\left(f \circ \sigma_{\diamond}^{n}(x)\right) *\left(f \circ \sigma_{\diamond}^{n}(y)\right)\right)\right) \\
& \leq \operatorname{Lip}\left(\sigma_{*}^{-2 n}\right) d\left(f \circ \sigma_{\diamond}^{n}(x \diamond y) * f \circ \sigma_{\diamond}^{n}\left(x \diamond y^{-1}\right), \sigma_{*}\left(\left(f \circ \sigma_{\diamond}^{n}(x)\right) *\left(f \circ \sigma_{\diamond}^{n}(y)\right)\right)\right) \\
& \leq \operatorname{Lip}\left(\sigma_{*}^{-2 n}\right) \varepsilon\left(\sigma_{\diamond}^{n}(x), \sigma_{\diamond}^{n}(y)\right)
\end{aligned}
$$

for all $x, y \in X$. Letting $n \rightarrow \infty$ in the last inequality, we get that $h$ satisfies (4).

Finally we complete the proof by showing the uniqueness. Assume that $h^{\prime}$ : $X \rightarrow Y$ is an arbitrary solution of (1) such that the mapping $x \mapsto d\left(f(x), h^{\prime}(x)\right)$ is bounded with bound in (4). Putting $x=y$ in (1), we have that

$$
h \circ \sigma_{\diamond}=\sigma_{*}^{2} \circ h, \quad h^{\prime} \circ \sigma_{\diamond}=\sigma_{*}^{2} \circ h^{\prime} .
$$

Using induction, we get

$$
\sigma_{*}^{-2 n} \circ h \circ \sigma_{\diamond}^{n}=h, \quad \sigma_{*}^{-2 n} \circ h^{\prime} \circ \sigma_{\diamond}^{n}=h^{\prime} .
$$


Hence, for every $x \in X$, we get by (4),

$$
\begin{aligned}
d\left(h^{\prime}(x), h(x)\right) & =d\left(\sigma_{*}^{-n} \circ h^{\prime} \circ \sigma_{\diamond}^{n}(x), \sigma_{*}^{-n} \circ h \circ \sigma_{*}^{n}(x)\right) \\
& \leq \operatorname{Lip}\left(\sigma_{*}^{-2 n}\right) d\left(h^{\prime} \circ \sigma_{\diamond}^{n}(x), h \circ \sigma_{*}^{n}(x)\right) \\
& \leq \operatorname{Lip}\left(\sigma_{*}^{-2 n}\right)\left[d\left(h^{\prime}\left(\sigma_{\diamond}^{n}(x)\right), f\left(\sigma_{*}^{n}(x)\right)\right)+d\left(f\left(\sigma_{\diamond}^{n}(x)\right), h\left(\sigma_{*}^{n}(x)\right)\right)\right] \\
& \left.\leq 2 \operatorname{Lip}\left(\sigma_{*}^{-2 n}\right) \sum_{k=0}^{\infty} \operatorname{Lip}\left(\sigma_{*}^{-2 k+2}\right) \varepsilon\left(\sigma_{\diamond}^{k}\right)\left(\sigma_{\diamond}^{n}(x)\right), \sigma_{\diamond}^{k}\left(\sigma_{\diamond}^{n}(x)\right)\right) \\
& \leq 2 \operatorname{Lip}\left(\sigma_{*}^{-2 n}\right) 2 \operatorname{Lip}\left(\sigma_{*}^{2 n}\right) \sum_{k=n}^{\infty} \operatorname{Lip}\left(\sigma_{*}^{-(2 k+2)}\right) \varepsilon\left(\sigma_{\diamond}^{k}(x), \sigma_{\diamond}^{k}(x)\right) .
\end{aligned}
$$

Since $\left\{\operatorname{Lip}\left(\sigma_{*}^{-2 n}\right) \operatorname{Lip}\left(\sigma_{*}^{2 n}\right)\right\}$ is bounded, taking the limit as $n \rightarrow \infty$ in the above inequality, we obtain $h^{\prime}(x)=h(x)$ for all $x \in X$. This completes the proof.

Theorem 2. Let $(X, \diamond)$ be a divisible square-symmetric groupoid and let $(Y, *, d)$ be a complete metric divisible square-symmetric groupoid. Assume that a mapping $f: X \rightarrow Y$ satisfies

$$
d\left(f(x \diamond y) * f\left(x \diamond y^{-1}\right), \sigma_{*}(f(x) * f(y))\right) \leq \varepsilon(x, y)
$$

for all $x, y \in X$ with $f\left(i d_{X}\right)=i d_{Y}$ and $\varepsilon: X^{2} \rightarrow[0, \infty)$ is a mapping such that

$$
L_{2}(x, y):=\sum_{n=1}^{\infty} \operatorname{Lip}\left(\sigma_{*}^{2 n}\right) \varepsilon\left(\sigma_{\diamond}^{-n}(x), \sigma_{\diamond}^{-n}(y)\right)<\infty
$$

for all $x, y \in X$. Then for every $x \in X$,

$$
h(x):=\lim _{n \rightarrow \infty} \sigma_{*}^{2 n} \circ f \circ \sigma_{\diamond}^{-n}(x)
$$

converges and $h: X \rightarrow Y$ is the solution of (1) such that

$$
d(f(x), h(x)) \leq \operatorname{Lip}\left(\sigma_{*}^{2}\right) L_{2}(x, x)
$$

for all $x \in X$.

Moreover, if $\left\{\operatorname{Lip}\left(\sigma_{*}^{-2 n}\right) \operatorname{Lip}\left(\sigma_{*}^{2 n}\right)\right\}$ is bounded then $h$ is the only solution of (1) such that the mapping $x \rightarrow d(h(x), f(x))$ is bounded with bound $L(x, y)$.

Proof. Define $f_{n}: X \rightarrow Y$ by

$$
f_{0}:=f, \quad f_{n}:=\sigma_{*}^{2 n} \circ f \circ \sigma_{\diamond}^{-n}
$$


for all positive integer $n \in \mathbb{N}$. Let $x \in X$ be fixed. Replacing $x$ and $y$ by $\sigma_{\diamond}^{-n}(x)$ in (7), we obtain

$$
d\left(f \circ \sigma_{\diamond}^{-n+1}(x), \sigma_{*}^{2} \circ f \circ \sigma_{\diamond}^{-n}(x)\right) \leq \varepsilon\left(\sigma_{\diamond}^{-n}(x), \sigma_{\diamond}^{-n}(x)\right) .
$$

Thus we figure out by (10)

$$
\begin{aligned}
d\left(f_{n-1}(x), f_{n}(x)\right) & =d\left(\sigma_{*}^{2 n-2} \circ f \circ \sigma_{\diamond}^{-n+1}(x), \sigma_{*}^{2(n-1)} \circ \sigma_{*}^{2} \circ f \circ \sigma_{\diamond}^{-n}(x)\right) \\
\leq & \operatorname{Lip}\left(\sigma_{*}^{2 n-2}\right) d\left(f \circ \sigma_{\diamond}^{-n+1}(x), \sigma_{*}^{2} \circ f \circ \sigma_{\diamond}^{-n+1}(x)\right) \\
\leq & \operatorname{Lip}\left(\sigma_{*}^{2 n-2}\right) \varepsilon\left(\sigma_{\diamond}^{-n}(x), \sigma_{\diamond}^{-n}(x)\right)
\end{aligned}
$$

for all $x \in X$. Hence, it follows by the above convergence that for all $n, m \in \mathbb{N}$

$$
\begin{aligned}
d\left(f_{n}(x), f_{n+m}(x)\right) & \leq \sum_{k=0}^{m-1} d\left(f_{n+k}(x), f_{n+k+1}(x)\right) \\
& \leq \sum_{k=0}^{m-1} \operatorname{Lip}\left(\sigma_{*}^{2(n+k)-2)}\right) \varepsilon\left(\sigma_{\diamond}^{-n-k}(x), \sigma_{\diamond}^{-n-k}(x)\right) \\
& \leq \sum_{k=n}^{n+m-1} \operatorname{Lip}\left(\sigma_{*}^{-2}\right) \operatorname{Lip}\left(\sigma_{*}^{2(k+1)}\right) \varepsilon\left(\sigma_{\diamond}^{-k}(x), \sigma_{\diamond}^{-k}(x)\right) \\
& \rightarrow 0 \text { as } n \rightarrow \infty .
\end{aligned}
$$

which shows that the sequence $\left\{f_{n}(x)\right\}$ is Cauchy in Y. Therefore, a mapping $h: X \rightarrow Y$ given by (8) is well defined. For all $n \in \mathbb{N}$, we have

$$
\begin{aligned}
d\left(f_{n+1}(x), f(x)\right) & \leq \sum_{k=0}^{n} d\left(f_{k+1}(x), f_{k}(x)\right) \\
& \leq \sum_{k=0}^{n} \operatorname{Lip}\left(\sigma_{*}^{2 k}\right) \varepsilon\left(\sigma_{\diamond}^{-k-1}(x), \sigma_{\diamond}^{-k-1}(x)\right) \\
& \leq \sum_{k=1}^{\infty} \operatorname{Lip}\left(\sigma_{*}^{2 k-2}\right) \varepsilon\left(\sigma_{\diamond}^{-k}(x), \sigma_{\diamond}^{-k}(x)\right) .
\end{aligned}
$$

Letting $n \rightarrow \infty$, we obtain (8) for the limit function $h$ of $\left\{f_{n}\right\}$.

Now we prove that $h$ satisfies (1). Putting $\sigma_{\diamond}^{-n}(x)$ and $\sigma_{\diamond}^{-n}(y)$ into $x$ and $y$ in (7) and using the endomorphism of $\sigma_{\diamond}^{n}$, we obtain

$$
\begin{aligned}
& d\left(f_{n}(x \diamond y) * f_{n}\left(x \diamond y^{-1}\right), \sigma_{*}\left(f_{n}(x) * f_{n}(y)\right)\right) \\
& =d\left(\left(\sigma_{*}^{2 n} \circ f \circ \sigma_{\diamond}^{-n}\right)(x \diamond y) *\left(\sigma_{*}^{2 n} \circ f \circ \sigma_{\diamond}^{-n}\right)\left(x \diamond y^{-1}\right),\right.
\end{aligned}
$$




$$
\begin{aligned}
& \left.\sigma_{*}\left(\sigma_{*}^{2 n} \circ f \circ \sigma_{\diamond}^{-n}(x)\right) *\left(\sigma_{*}^{2 n} \circ f \circ \sigma_{\diamond}^{-n}(y)\right)\right) \\
= & d\left(\left(\sigma_{*}^{2 n} \circ f \circ \sigma_{\diamond}^{-n}\right)(x \diamond y) *\left(\sigma_{*}^{2 n} \circ f \circ \sigma_{\diamond}^{-n}\right)\left(x \diamond y^{-1}\right),\right. \\
& \left.\sigma_{*}^{2 n+1}\left(f \circ \sigma_{\diamond}^{-n}(x)\right) *\left(f \circ \sigma_{\diamond}^{-n}(y)\right)\right) \\
\leq & \operatorname{Lip}\left(\sigma_{*}^{2 n}\right) d\left(\left(f \circ \sigma_{\diamond}^{-n}\right)(x \diamond y) *\left(f \circ \sigma_{\diamond}^{-n}\right)\left(x \diamond y^{-1}\right),\right. \\
& \sigma_{*}\left(f \circ \sigma_{\diamond}^{-n}(x) * f \circ \sigma_{\diamond}^{-n}(y)\right) \\
\leq & \operatorname{Lip}\left(\sigma_{*}^{2 n}\right) \varepsilon\left(\sigma_{\diamond}^{-n}(x), \sigma_{\diamond}^{-n}(y)\right)
\end{aligned}
$$

Whence, taking the limit as $n \rightarrow \infty$ in the last inequality, we get that $f$ is a homomorphism, that is, it satisfies (1). The proof of the uniqueness of $h$ is now based on the identity

$$
\sigma_{*}^{2 n} \circ h \circ \sigma_{\diamond}^{-n}=h, \quad \sigma_{*}^{2 n} \circ h^{\prime} \circ \sigma_{\diamond}^{-n}=h^{\prime} .
$$

In fact, let $\left\{\operatorname{Lip}\left(\sigma_{*}^{2 n}\right) \operatorname{Lip}\left(\sigma_{*}^{-2 n}\right)\right\}$ be bounded and assume that $f^{\prime}$ is another solution of (1) such that the mapping $x \mapsto d\left(f(x) \cdot h^{\prime}(x)\right)$ is bounded with bound (9). Then for every $x \in X$ we get by (9) and (10)

$$
\begin{aligned}
d\left(h^{\prime}(x), h(x)\right) & =d\left(\sigma_{*}^{2 n} \circ h^{\prime} \circ \sigma_{\diamond}^{-n}(x)\right) \\
& \left.\leq \operatorname{Lip}\left(\sigma_{*}^{2 n}\right) d\left(h^{\prime} \circ \sigma_{\diamond}^{-n}\right), h \circ \sigma_{\diamond}^{-n}\right) \\
& \left.\left.\leq \operatorname{Lip}\left(\sigma_{*}^{2 n}\right)\left[d\left(h^{\prime} \circ \sigma_{\diamond}^{-n}\right), h \circ \sigma_{\diamond}^{-n}\right)+d\left(f \circ \sigma_{\diamond}^{-n}\right), h \circ \sigma_{\diamond}^{-n}\right)\right] \\
& \leq 2 \operatorname{Lip}\left(\sigma_{*}^{2 n}\right) \sum_{k=1}^{\infty} \operatorname{Lip}\left(\sigma_{*}^{2 k}\right) \varepsilon\left(\sigma_{\diamond}^{-k}\left(\sigma_{\diamond}^{-n}(x)\right), \sigma_{\diamond}^{-k}\left(\sigma_{\diamond}^{-n}(x)\right)\right) \\
& \left.\leq 2 \operatorname{Lip}\left(\sigma_{*}^{2 n}\right) \operatorname{Lip}\left(\sigma_{*}^{-2 n}\right) \sum_{k=1}^{\infty} \operatorname{Lip}\left(\sigma_{*}^{2 n+2 k}\right) \varepsilon\left(\sigma_{\diamond}^{-n-k}(x)\right), \sigma_{\diamond}^{-n-k}(x)\right) \\
& \left.\leq 2 \operatorname{Lip}\left(\sigma_{*}^{2 n}\right) \operatorname{Lip}\left(\sigma_{*}^{-2 n}\right) \sum_{k=n}^{\infty} \operatorname{Lip}\left(\sigma_{*}^{2 k}\right) \varepsilon\left(\sigma_{\diamond}^{-k}(x)\right), \sigma_{\diamond}^{-k}(x)\right) \\
& \rightarrow 0 \quad \text { as } \quad n \rightarrow \infty,
\end{aligned}
$$

since $\left\{\operatorname{Lip}\left(\sigma_{*}^{2 n}\right) \operatorname{Lip}\left(\sigma_{*}^{-2 n}\right)\right\}$ is bounded. This proves the uniqueness of $h$. The proof of the theorem is complete.

\section{The Hyers-Ulam Stability of Functional Equation (1) via Fixed Point Theorem}

In this section, we will mention about the stability of the functional equation

(1) using another method. First, we give the definition of a generalized metric 
on a set $X$. A function $d: X \times X \rightarrow[0, \infty]$ is called a generalized metric on $X$ if $d$ satisfies the following properties

(1) $d(x, y)=0$ if and only if $x=y$;

(2) $d(x, y)=d(y, x)$ for all $x, y \in X$;

(3) $d(x, z) \leq d(x, y)+d(y, z)$ for all $x, y, z \in Y$.

In [13], Margolis and Diaz constructed the variable method using a fixed point theory, which is variously applied to the theory of functional equations.

Theorem 3. (The Alternative Fixed Point [13]) Let $(X, d)$ be a complete generalized metric space and let $J: X \rightarrow X$ be a strictly contractive mapping with Lipschitz constant $L<1$. Then for each given element $x \in X$, either $d\left(J^{n} x, J^{n+1} x\right)=\infty$ for all nonnegative integers $n$ or there exists a positive integer $n_{0}$ such that

(1) $d\left(J^{n} x, J^{n+1} x\right)<\infty, \forall n \geq n_{0}$

(2) the sequence $\left\{J^{n} x\right\}$ converges to a fixed point $y^{*}$ of $J$;

(3) $y^{*}$ is the unique fixed point of $J$ in the set $Y=\left\{y \in X \mid d\left(J^{n_{0}} x, y\right)<\infty\right\}$;

(4) $d\left(y, y^{*}\right) \leq \frac{1}{1-L} d(y, J y)$ for all $y \in Y$.

Using the alternative fixed point theorem, we investigate the stability of the functional equation (1).

Theorem 4. Let $(X, \diamond)$ be a divisible square-symmetric groupoid and let $(Y, *, d)$ be a complete metric divisible square-symmetric groupoid and $L \in$ $(0,1)$. Assume that a mapping $f: X \rightarrow Y$ satisfies

$$
d\left(f(x \diamond y) * f\left(x \diamond y^{-1}\right), \sigma_{*}(f(x) * f(y))\right) \leq \varepsilon(x, y)
$$

for all $x, y \in X$ with $f\left(i d_{X}\right)=i d_{Y}$ and $\varepsilon: X^{2} \rightarrow[0, \infty)$ is a mapping such that

$$
\varepsilon\left(\sigma_{\diamond}(x), \sigma_{\diamond}(y)\right) \leq \frac{L}{\operatorname{Lip}\left(\sigma_{*}^{-2}\right)} \varepsilon(x, y)
$$

for all $x, y \in X$. Then for every $x \in X$,

$$
H(x):=\lim _{n \rightarrow \infty} \sigma_{*}^{-2 n} \circ f \circ \sigma_{\diamond}^{n}(x)
$$


converges and $H: X \rightarrow Y$ is the solution of (1) such that

$$
d(f(x), H(x)) \leq \frac{\operatorname{Lip}\left(\sigma_{*}^{-2}\right)}{1-L} \varepsilon(x, x)
$$

for all $x \in X$.

Proof. Consider the set

$$
S:=\{g: X \rightarrow Y\}
$$

and introduce a generalized metric on $S$ :

$$
d^{\prime}(g, h):=\inf \{c>0: d(g(x), h(x)) \leq c \cdot \varepsilon(x, x)\}
$$

where, as usual, inf $\emptyset=+\infty$. It is easy to show that $\left(S, d^{\prime}\right)$ is complete (see the proof of Lemma 2.1 of [14])

Now we consider the linear mapping $T: S \rightarrow S$ such that

$$
T g:=\sigma_{*}^{-2} \circ g \circ \sigma_{\diamond}
$$

for all $x \in X$.

Let $g, h \in S$ be given such that $d^{\prime}(g, h)=c$. Then

$$
\begin{aligned}
d(T g(x), T k(x)) & =d\left(\sigma_{*}^{-2} \circ g \circ \sigma_{\diamond}(x), \sigma_{*}^{-2} \circ k \circ \sigma_{\diamond}(x)\right) \\
& \leq \operatorname{Lip}\left(\sigma_{*}^{-2}\right) d\left(f \circ \sigma_{\diamond}(x), k \circ \sigma_{\diamond}(x)\right) \\
& \leq \operatorname{Lip}\left(\sigma_{*}^{-2}\right) c \varepsilon\left(\sigma_{\diamond}(x), \sigma_{\diamond}(x)\right) \\
& \leq c \cdot \operatorname{L\varepsilon }(x, x)
\end{aligned}
$$

for all $x \in X$. So $d^{\prime}(g, h)=c$ implies that $d^{\prime}(T g, T h) \leq L c$. This means that

$$
d^{\prime}(T g, T h) \leq L d^{\prime}(g, h)
$$

for all $g, h \in S$.

Letting $y=x$ in (11), we get

$$
d\left(f\left(\sigma_{\diamond}(x)\right), \sigma_{*}^{2}(f(x))\right) \leq \varepsilon(x, x)
$$

for all $x \in X$. So

$$
\left.d\left(f(x), \sigma_{*}^{-2} \circ f \circ \sigma_{\diamond}(x)\right)\right) \leq \operatorname{Lip}\left(\sigma_{*}^{-2}\right) \varepsilon(x, x)
$$

for all $x \in X$. So $d^{\prime}(f, T f) \leq \operatorname{Lip}\left(\sigma_{*}^{-2}\right)$. 
By Theorem 3, there exists a mapping $H: X \rightarrow Y$ satisfying the following (1) $H$ is a fixed point of $T$, i.e.,

$$
H\left(\sigma_{\diamond}(x)\right)=\sigma_{*}^{2}(H(x))
$$

for all $x \in X$. The mapping $H$ is a unique fixed point of $T$ in the set

$$
M=\{g \in S: d(f, g)<\infty\} .
$$

This means that $H$ is a unique fixed point satisfying (14).

(2) $d^{\prime}\left(T^{n} f, H\right) \rightarrow 0$ as $n \rightarrow \infty$. This implies the equality

$$
\lim _{n \rightarrow \infty} \sigma_{*}^{-2 n} \circ f \circ \sigma_{\diamond}^{n}(x)=H(x)
$$

for all $x \in X$.

(3) $d^{\prime}(f, H) \leq \frac{1}{1-L} d^{\prime}(f, T f) \leq \frac{\operatorname{Lip}\left(\sigma_{*}^{-2}\right)}{1-L}$, which implies the inequality

$$
d(f(x), H(x)) \leq \frac{\operatorname{Lip}\left(\sigma_{*}^{-2}\right)}{1-L} \varepsilon(x, x)
$$

for all $x \in X$. This implies that the inequality (14) holds.

By (12),

$$
\begin{aligned}
& d\left(\left(\sigma_{*}^{-2 n} \circ f \circ \sigma_{\diamond}^{n}(x \diamond y)\right) *\left(\sigma_{*}^{-2 n} \circ f \circ \sigma_{\diamond}^{n}\left(x \diamond y^{-1}\right)\right),\right. \\
& \left.\quad \sigma_{*}\left(\left(\sigma_{*}^{-2 n} \circ f \circ \sigma_{\diamond}^{n}(x)\right) *\left(\sigma_{*}^{-2 n} \circ f \circ \sigma_{\diamond}^{n}(y)\right)\right)\right) \\
& \leq \operatorname{Lip}\left(\sigma_{*}^{-2 n}\right) \varepsilon\left(\sigma_{\diamond}^{n}(x), \sigma_{\diamond}^{n}(y)\right) \\
& \leq L^{n} \varepsilon(x, y)
\end{aligned}
$$

for all $x, y \in X$ and $n \in \mathbb{N}$. Taking $n \rightarrow \infty$, we get $H$ satisfies (1). This completes the proof.

Theorem 5. Let $(X, \diamond)$ be a divisible square-symmetric groupoid and let $(Y, *, d)$ be a complete metric divisible square-symmetric groupoid and $L \in$ $(0,1)$. Assume that a mapping $f: X \rightarrow Y$ satisfies

$$
d\left(f(x \diamond y) * f\left(x \diamond y^{-1}\right), \sigma_{*}(f(x) * f(y))\right) \leq \varepsilon(x, y)
$$

for all $x, y \in X$ with $f\left(i d_{X}\right)=i d_{Y}$ and $\varepsilon: X^{2} \rightarrow[0, \infty)$ is a mapping such that

$$
\varepsilon\left(\sigma_{\diamond}^{-1}(x), \sigma_{\diamond}^{-1}(y)\right) \leq \frac{L}{\operatorname{Lip}\left(\sigma_{*}^{2}\right)} \varepsilon(x, y)
$$


for all $x, y \in X$. Then for every $x \in X$,

$$
H(x):=\lim _{n \rightarrow \infty} \sigma_{*}^{-2 n} \circ f \circ \sigma_{\diamond}^{n}(x)
$$

converges and $H: X \rightarrow Y$ is the solution of (1) such that

$$
d(f(x), H(x)) \leq \frac{L}{\operatorname{Lip}\left(\sigma_{*}^{2}\right)(1-L)} \varepsilon(x, x)
$$

for all $x \in X$.

Proof. Putting $y=x$ in (15), we get

$$
d\left(f\left(\sigma_{\diamond}(x)\right), \sigma_{*}^{2} f(x)\right) \leq \varepsilon(x, x)
$$

for all $x \in X$. If we set $\left(S, d^{\prime}\right)$ which is introduced in Theorem 4 and define the operator $T$ such that

$$
\operatorname{Tg}(x):=\sigma_{*}^{2} \circ f \circ \sigma_{\diamond}^{-1}(x)
$$

for all $g \in S$ and all $x \in X$. The rest proof is similar to that of Theorem 4 .

\section{References}

[1] J. Aczel and J. Dhombres, Functional Equations in Several Variables, Cambridge University Press, 1989, doi: 10.1017/CBO9781139086578.

[2] T. Aoki, On the stability of the linear transformation in Banach spaces, J. Math. Soc. Japan, 2, No. 1-2 (1950), 64-66, doi: 10.2969/jmsj/00210064.

[3] D.G. Bourgin, Classes of transformations and bordering transformations, Bull. Amer. Math. Soc., 57 (1951), 223-237, doi: 10.1090/S0002-9904-1951-09511-7.

[4] I.-S. Chang, H.-M. Kim, On the Hyers-Ulam stability of quadratic functional equations, J. Inequal. App., 3, No. 3 (2002), 1-12.

[5] Y-J. Cho, Th. M. Rassias, R. Saadati, Stability of Functional Equations in Random Normed Spaces, Springer Optimization and Its Applications, 86, Springer, 2013, doi: 10.1007/978-1-4614-8477-6.

[6] S. Czerwik, On the stability of the quadratic mapping in normed spaces, Bull. Abh. Math. Sem. Univ. Hamburg, 62 (1992), 59-64, doi: 10.1007/BF02941618.

[7] S. Czerwik, Stability of Functional Equations of Ulam-Hyers-Rassias Type, Hadronic Press, Florida, 2003.

[8] P. Găvruta, A generalization of the Hyers-Ulam-Rassias stability of approximately additive mappings, J. Math. Anal. Appl., 184 (1994), 431-436.

[9] D. H. Hyers, On the stability of the linear functional equation, Proc. Nat. Acad. Sci., 27 (1941), 222-224, doi: 10.1073/pnas.27.4.222. 
[10] S-M. Jung, Hyers-Ulam-Rassias Stability of Functional Equations in Nonlinear Analysis, Springer Optimization and Its Applications, USA, 2011, doi: 10.1007/978-1-4419-9637-4.

[11] G. H. Kim, Addendum to 'On the stability of functional equations on square-symmetric groupoid, J. Nonlinear Anal, 62 (2006), 365-381.

[12] H.-M. Kim, H.-Y. Shin, Generalized Hyers-Ulam stability of refined quadratic functional equations, Inter. J. Pure Appl. Math, 98, No. 1 (2015), 65-79.

[13] B. Margolis, J.B. Diaz, A fixed point theorem of the alternative for contractions on a generalized complete metric space, Bull. Amer. Math. Soc., 126 (1968), 305-309.

[14] D. Mihet, V. Radu, On the stability of the additive Cauchy functional equation in random normed spaces, J. Math. Anal. Apple., 343 (2008), 567-572.

[15] M. Mirzavaziri and M. S. Moslehian, A fixed point approach to stability of a quadratic equation, Bull. Brazilian Math. Soc., 37 (2006), 361-376, doi: 10.1007/s00574-006-0016$\mathrm{z}$.

[16] Th. M. Rassias, Functional Equations, Inequalities and Applications, Kluwer Academic Publishers, Dordrecht, Boston and London (2003), doi: 10.1007/978-94-017-0225-6.

[17] Th. M. Rassias, On the stability of the linear mapping in Banach spaces, Proc. Amer. Math. Soc., 72 (1978), 297-300, doi: 10.1090/S0002-9939-1978-0507327-1.

[18] F. Skof, Local properties and approximations of operators, Rend. Sem. Math. Fis. Milano, 53 (1983), 113-129.

[19] S. M. Ulam, Problems in Modern Mathematics, Chapter 6, Wiley Interscience, New York, 1964. 
